\title{
Cytoplasmic and stromal expression of laminin $\gamma 2$ chain correlates with infiltrative invasion in ovarian mucinous neoplasms of gastro-intestinal type
}

\author{
EMI OKUMA ${ }^{1}$, YOSHIHIRO OHISHI ${ }^{1,3}$, YOSHINAO ODA $^{1}$, SHINICHI AISHIMA $^{1}$, \\ SHUICHI KURIHARA ${ }^{1}$, IZUMI NISHIMURA ${ }^{1}$, MASAFUMI YASUNAGA ${ }^{1}$, \\ HIROAKI KOBAYASHI ${ }^{2}$, NORIO WAKE ${ }^{2}$ and MASAZUMI TSUNEYOSHI ${ }^{1}$
}

\begin{abstract}
Departments of ${ }^{1}$ Anatomic Pathology, and ${ }^{2}$ Gynecology and Obstetrics, Kyushu University Hospital, Fukuoka; ${ }^{3}$ Department of Diagnostic Laboratory, Kyushu University Hospital at Beppu, Beppu, Japan
\end{abstract}

Received April 21, 2010; Accepted June 2, 2010

DOI: 10.3892/or_00001019

\begin{abstract}
Ovarian mucinous neoplasms of gastro-intestinal type (GI-type) are known to be a heterogeneous tumor composed of benign, borderline and non-invasive and invasive malignant lesions. The presence of infiltrative invasion is also known to be an important prognostic factor of this neoplasm. Laminin $\gamma 2$ chain, known to stimulate tumor cell invasion and migration, has not been sufficiently investigated in ovarian mucinous neoplasms. The purpose of this study was thus to clarify the role of laminin $\gamma 2$ in ovarian mucinous neoplasms of GI-type. We selected each morphological phase of tumor development from 61 cases of mucinous neoplasms of the GI-type: 55 adenoma lesions, 60 borderline lesions, 20 microinvasive lesions, 17 intraepithelial carcinoma lesions, 38 expansile invasive carcinoma lesions, 19 infiltrative invasive carcinoma lesions and 5 mural nodules lesions; and evaluated the localization of laminin $\gamma 2$ in the lesions using immunohistochemical method. The staining pattern was classified into i) basement membranous (BM), ii) cytoplasmic (CYT) and iii) stromal (S) pattern. The BM pattern was characteristic in adenoma, borderline, and interaepithelial and expansile invasive carcinoma lesions. The CYT and S patterns were characteristic in infiltrative invasive lesions. The staining pattern of mural nodules was similar to that of infiltrative invasion. The infiltrative invasion of GI-type ovarian mucinous neoplasms may be promoted by cytoplasmic and/or stromal expression of laminin $\gamma 2$ chain.
\end{abstract}

Correspondence to: Dr Yoshihiro Ohishi, Department of Anatomic Pathology, Kyushu University Hospital, Maidashi 3-1-1, Higashi-ku, Fukuoka 812-8582, Japan

E-mail: yohishi@surgpath.med.kyushu-u.ac.jp

Key words: laminin $\gamma 2$ chain, ovarian mucinous neoplasms of gastro-intestinal type, infiltrative invasion, mural nodule

\section{Introduction}

Ovarian mucinous neoplasms of the GI-type account for 31\% of all ovarian surface-stromal tumors (1) and are known to be heterogeneously composed of benign, borderline, invasive and even undifferentiated (anaplastic) carcinoma within a single tumor (2-4). This has led to basic agreement that GItype invasive mucinous carcinoma may develop from mucinous adenoma through borderline tumor and noninvasive carcinoma. Because of the heterogeneity, extensive microscopic sampling is necessary for an accurate histological diagnosis of these tumors.

The GI-type mucinous ovarian tumor has recently been subclassified into the following categories: adenoma, borderline tumor with or without intraepithelial carcinoma, microinvasion, expansile invasive carcinoma, and infiltrative invasive carcinoma $(2,4-7)$. It is generally agreed that nuclear atypia and stromal invasion are key morphological features in the tumor progression $(2,4-6,8)$, and the destructive invasion, which is characterized by infiltrative growth pattern, is considered one of the most important adverse prognostic factors $(2,3,6,7)$.

Mucinous tumor with mural nodule exhibits one or more discrete well-circumscribed nodules protruding into the lumen of a cystic ovarian mucinous neoplasm. These nodules present a distinctly different microscopic appearance from the mucinous epithelial component, revealing anaplastic carcinoma, sarcoma, sarcomatoid carcinoma or sarcoma-like features (9-13). The mucinous epithelial component can be carcinoma, borderline tumor or adenoma. Although the sarcoma-like feature is not a neoplastic feature but instead a reactive tumor-like lesion that is known to have a favorable clinical course, true sarcomatoid or carcinomatous mural nodules are often in an advanced clinical stage at the primary operation, and exhibit aggressive biological behavior.

Laminin-5, the multifunctional cell adhesive molecules, is composed of $\alpha 3, \beta 3$ and $\gamma 2$ chains. It is a major component of the basement membrane of various types of epithelial tissue and plays important roles in cell differentiation, adhesion, and migration $(14,15)$. Pyke et al $(4,16)$ demonstrated that laminin-5 is frequently localized in the cytoplasm of invading 
cancer cells, which suggests that laminin-5 may be involved in tumor invasion. Overexpression of laminin $\gamma 2$ chain has been particularly demonstrated on the invasive front of various carcinoma, including colorectal $(16,17)$, gastric (18), intrahepatic cholangial (19), lung (20), breast (16), and pancreatic (21) adenocarcinomas, and head and neck (22), esophageal (23) and uterine cervical $(16,17,24)$ squamous cell carcinomas and malignant melanomas (23). However, the relationship between laminin $\gamma 2$ chain expression and ovarian GI-type mucinous neoplasms, including mural nodules, has not been elucidated. We therefore investigated the association between laminin $\gamma 2$ localization and invasive pattern of GI-type ovarian mucinous neoplasms.

\section{Materials and methods}

Tissue specimens. We retrieved all cases registered as mucinous adenocarcinoma or mucinous borderline tumor and mucinous cystic tumor with mural nodules in the Department of Anatomic Pathology, Graduate School of Medical Science, Kyushu University between 1982 and 2007, Kyushu Welfare Hospital between 1998 and 2006, and Fukuoka Red Cross Hospital between 2002 and 2006. Endocervical-like mucinous borderline neoplasms and mucinous tumors associated with pseudomyxoma peritonei were excluded. We also excluded mucinous tumors containing a teratomatous component or Brenner tumor. To rule out metastatic cases, we excluded cases which have malignant tumor in the gastro-intestinal tract or breast or endometrium in reference to clinical information. We also excluded the bilateral tumors of any size and unilateral tumors with size $<10 \mathrm{~cm}$, according to the previous studies $(7,25,26)$.

Five cases of mural nodule and 44 cases of mucinous adenocarcinoma were selected. Twelve cases of mucinous borderline tumor (MBT) were also randomly selected. All available slides of these 61 cases were reviewed. These 61 cases contained a number of morphological phase of mucinous tumor development in an individual case (such as, a carcinoma cases also contain each of the lesions, adenoma, borderline and intraepithelial carcinoma), and we extracted each lesion from 61 cases. The selected lesions were classified into seven histological categories based on the following definition.

Definition of histological classification for selected lesions. i) Adenoma. Multicystic lesions lined by a single layer of tall columnar epithelium without nuclear atypia.

ii) Borderline. Multicystic lesions lined by stratified, proliferative gastrointestinal-type mucinous epithelium exhibiting tufted and papillary intraglandular growth with mild (grade 1) or only focally moderate (grade 2) nuclear atypia (Fig. 1A). iii) Intraepithelial carcinoma. Lesions with severe (grade 3) nuclear atypia associated with above-mentioned borderline lesions lacking stromal invasion (Fig. 1C).

iv) Expansile invasive carcinoma. Invasive mucinous carcinoma assigned by a complex or confluent arrangement of malignant glands and papillae exhibiting diffuse moderate (grade 2) to severe (grade 3) nuclear atypia without demonstrable destructive (infiltrative) invasion (Fig. 1E). The expansile growth pattern had to be at least $3 \mathrm{~mm}$ in each of two linear dimensions to qualify as invasive carcinoma. v) Microinvasion. Small foci (less than $3 \mathrm{~mm}$ in greatest linear dimension) of stromal invasion characterized by single cells, glands, or small clusters/nests with or without nuclear atypia. We subdivided the microinvasive foci into two types: a) a well differentiated form of microinvasion, characterized by invasive cells forming sharp outlined glands (Fig. 2A), b) a poorly differentiated form of microinvasion, characterized by invasive small nests or single cells (Fig. 2C).

vi) Infiltrative invasive carcinoma. Characterized by obvious destructive stromal invasion exceeding microinvasion, in the form of small nests, irregular glands, cell clusters or single cells disorderly infiltrating the stroma and sometimes eliciting a desmoplastic stromal reaction. We divided infiltrative invasion into two subcategories: a) a well differentiated form of infiltrative invasion (Fig. 3A), b) a poorly differentiated form of infiltrative invasion (Fig. 3C), as was done with microinvasion.

vii) Mural nodules. Defined above in the introduction section (Fig. 4A and C). Nuclear grade was evaluated in all cases as mild (grade 1), moderate (grade 2), or severe (grade 3 ), considering nuclear size, nuclear hyperchromasia and the prominence of nucleoli.

Based on the most aggressive morphological lesion within a tumor, we diagnosed and classified the cases as follows: 7 cases of MBT, 2 cases of MBT with intraepithelial carcinoma, 3 cases of MBT with microinvasion, 29 cases of expansile invasive carcinoma, 15 cases of infiltrative invasive carcinoma and 5 cases of mural nodules. The number of sections per tumor ranged from 4 to 60 (mean 17.9; median 16). The mean numbers per centimeter of tumor diameter available for review was 1.1. Clinical and pathological information regarding patient's age, presentation, tumor size, stage, treatment and follow-up data, when available, was obtained from the clinical records or tumor registers as well as from written communication from pathologists and gynecologists.

Immunohistochemistry. Immunohistochemistry was performed according to the peroxidase-labeled streptavidinbiotin technique method using a Histofine streptavidinbiotin-peroxidase kit (Nichirei, Tokyo, Japan). After the endogenous peroxidase activity was blocked by methanol containing $0.3 \%$ hydrogen peroxide, the sections staining for laminin $\gamma 2$ were treated with Protease XXIV (Sigma, St. Louis, MO, USA) at room temperature. The primary antibody used in this study was mouse monoclonal antibody against the laminin $\gamma 2$ chain (clone D4B5, dilution 1:100; Chemicon, Temacula, CA, USA). The antibody against laminin $\gamma 2$ chain was developed against amino-acid residues 382-608 of the human laminin $\gamma 2$ chain, and this antibody recognizes the 150- and 105-kDa chain proteins (18).

Immunohistochemical evaluation. Localization of the laminin $\gamma 2$ chain was divided into three patterns: staining of basement membrane (BM), staining of tumor cytoplasm (CYT), staining of the stroma around the tumor cells (S). We selected the strongest stained area per each expression pattern at low magnification. Then, the areas were viewed with $10 \mathrm{x}$ objective, and laminin $\gamma 2$ expression was graded on a scale 0-2 as follows: 0, either no positive tumor cells present or only a few positive tumor cells observed; 1 , laminin $\gamma 2$ 

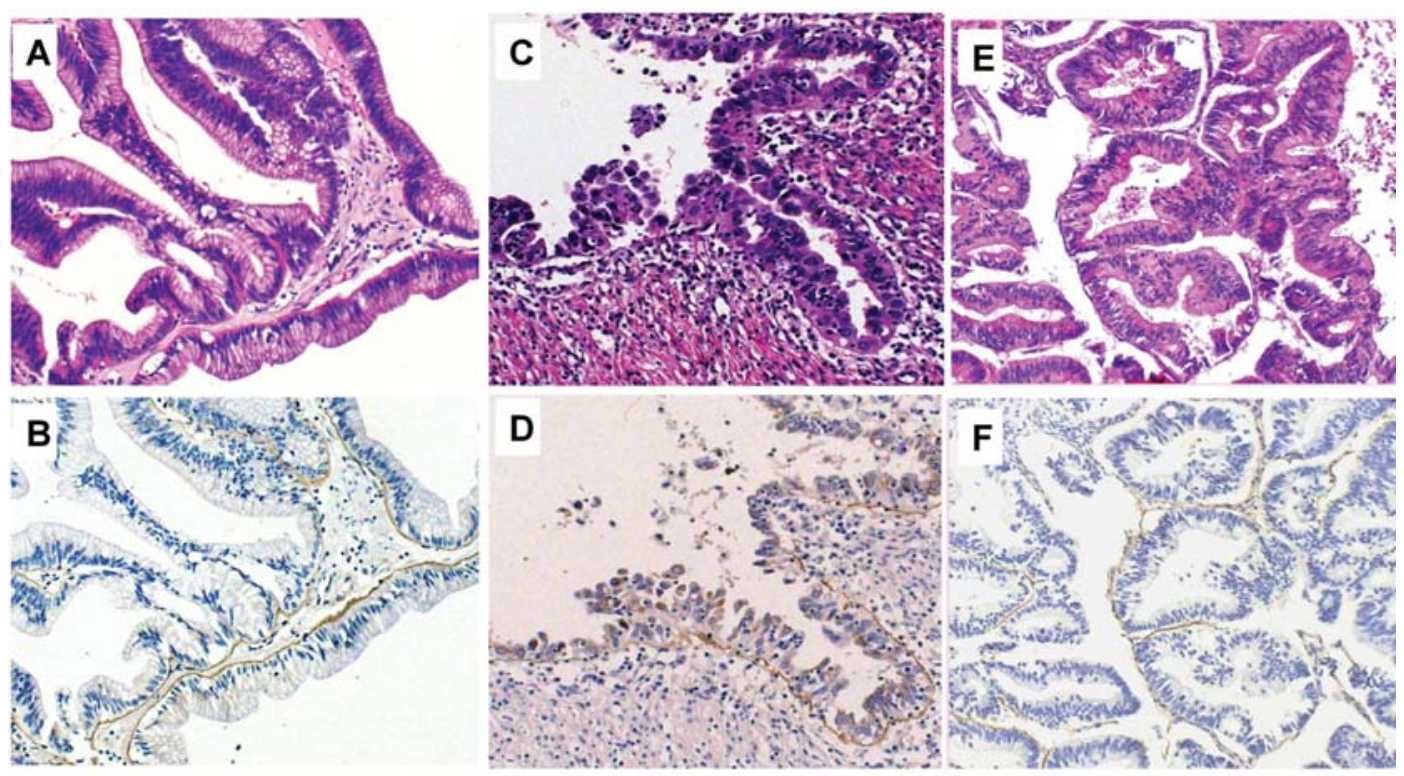

Figure 1. Hematoxylin-eosin (HE) findings and laminin $\gamma 2$ immnohistochemical results of non-obviously invasive lesions. (A) Borderline lesion, HE, x100. (B) Borderline lesion, laminin $\gamma 2$ chain immunostaining, x100; the score is BM 2. (C) Intraepithelial carcinoma lesion, HE, x100. (D) Intraepithelial carcinoma lesion, laminin $\gamma 2$ chain immunostaining, $x 100$; the score is BM 2. (E) Expansile invasive lesion, HE, x100. (F) Expansile invasive lesion, laminin $\gamma 2$ chain immunostaining, $\mathrm{x} 100$; the basement membrane staining is slightly weakened and discontinuous compared to that of borderline lesion, the score is BM 1 .

positive tumor cells were accounted for $<30 \%$ of the tumor cells within the visual field; 2 , laminin $\gamma 2$ positive tumor cells were accounted for $\geq 30 \%$ of the tumor cells within the visual field (20).

Statistics. The $\chi^{2}$ test was used to evaluate the association between histological appearance and three expression pattern of laminin $\gamma$ 2. The results were considered significant at $\mathrm{P}<0.05$. Analyses were done using Microsoft excel and Statcel 2 (oms-publishing) and JMP 7.

\section{Results}

Histological review of the 61 cases of the ovarian mucinous neoplasms. After the review of hematoxylin-eosin stained slides in the 61 cases, we extracted 55 adenoma lesions, 60 borderline tumor lesions, 17 intraepithelial carcinoma lesions, 19 microinvasive lesions, 38 expansile invasive carcinoma lesions, 19 infiltrative invasive carcinoma lesions and 5 mural nodules. Out of 19 microinvasive lesions, 18 well differentiated lesions and 6 poorly differentiated lesions were identified. Both well and poorly differentiated lesions were identified in 5 cases. Similarly, out of 19 infiltrative invasive carcinoma lesions, there were 12 well differentiated and 15 poorly differentiated lesions. Both types of invasion were identified in 8 cases.

All mural nodules in this study were composed of anaplastic or sarcomatoid carcinoma. Anaplastic carcinoma was found in 4 cases. It is composed of polygonal or relatively rounded carcinoma cells with pleomorphic nuclei and eosinophilic cytoplasm arranged in disorderly solid sheets. Myxoid, edematous, or hyalinized stroma often surrounded the carcinoma cells. Sarcomatoid carcinoma, characterized by a proliferation of spindle cells with hyperchromatic, and enlarged nuclei and moderately abundant cytoplasm arranged in fascicles were detected in all 5 cases. Rhabdoid cells were detected in 3 cases. In 3 out of 5 cases, infiltrative invasive mucinous adenocarcinoma was located at the transition zone between the mural nodule and non-invasive mucinous epithelium. In the remaining 2 cases, infiltrative invasive adenocarcinoma could not be identified; one case was associated with expansile invasive carcinoma and the other case was associated with MBT with microinvasion. The epithelial nature of the anaplastic/sarcomatoid carcinoma components was confirmed by immunohistochemistry (IHC) of EMA and AE1/AE3 at the initial diagnosis.

Correlation between morphological pattern and the immunohistochemical results of laminin $5 \gamma 2$ chain. The IHC results according to the each morphological lesion are summarized in Table I, and comparisons of laminin $\gamma 2$ expression between non-obviously invasive lesions (adenoma, borderline without microinvasion, intraepithelial carcinoma and expansile invasive carcinoma) and that of obviously invasive lesions (infiltrative invasive carcinoma, anaplastic carcinoma and sarcomatoid carcinoma) are summarized in Table II. Comparisons of laminin $\gamma 2$ expression between well and poorly differentiated lesions in infiltrative invasion and microinvasion respectively are also summarized in Table II. $\mathrm{BM}$ staining was frequently seen in non-obviously invasive lesions. In contrast, CYT and S staining were frequently seen in obviously invasive lesions. In non-obviously invasive lesions, the intensity of BM staining became slightly weakened and discontinuous as the tumor progressed from benign to borderline to carcinoma (Figs. 1B, D and F).

Among invasive carcinoma, well differentiated lesions expressed BM staining more frequent than poorly differentiated lesions. On the contrary, CYT staining was more frequent in poorly differentiated lesions than in well differentiated lesions. There was no significant difference in $\mathrm{S}$ staining 

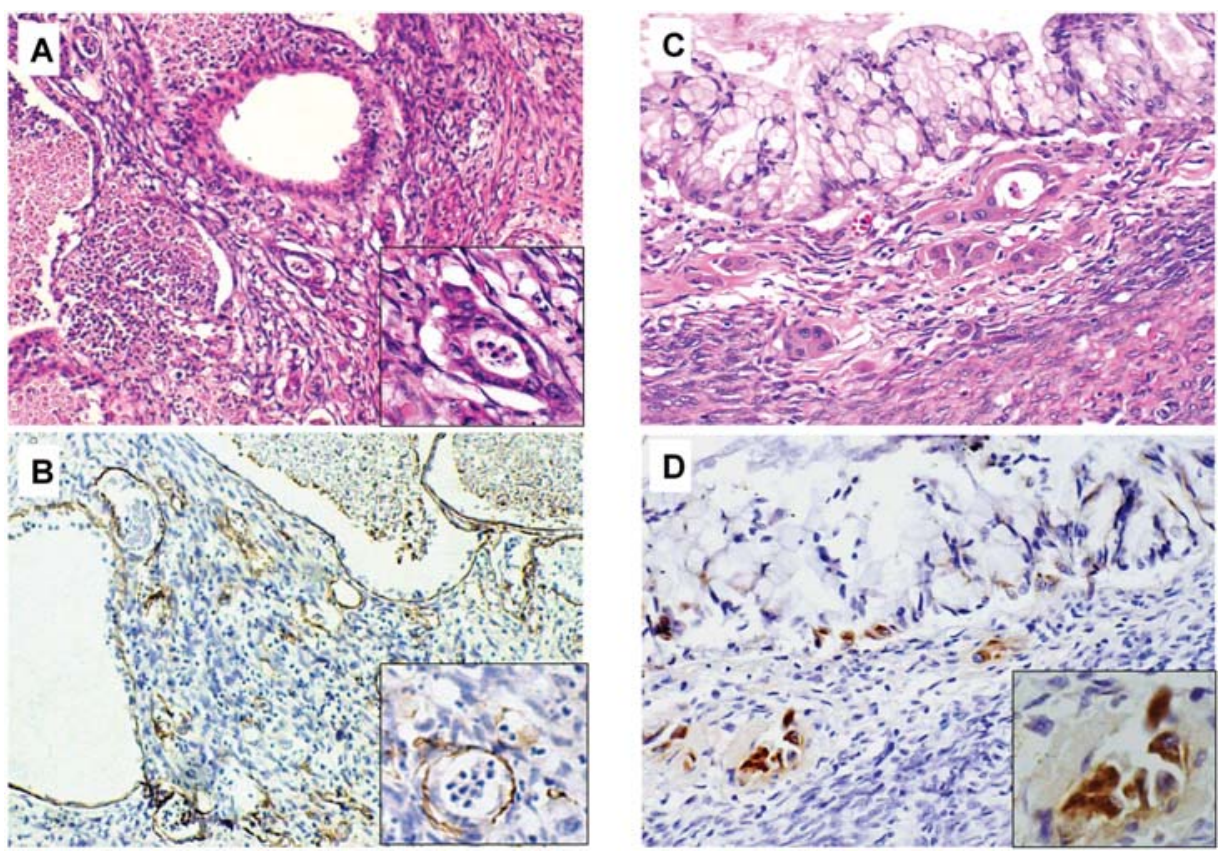

Figure 2. Hematoxylin-eosin findings and laminin $\gamma 2$ imunohistochemical results of microinvasion. (A) Well differentiated microinvasive lesion, HE, x100. (B) Well differentiated microinvasive lesion, laminin $\gamma 2$ chain immunostaining, x100; the score is BM 2. (C) Poorly differentiated micro-invasive lesion, HE, $\mathrm{x} 100$. (D) Poorly differentiated micro-invasive lesion, laminin $\gamma 2$ chain immunostaining, $\mathrm{x} 100$; the score is CYT 2.
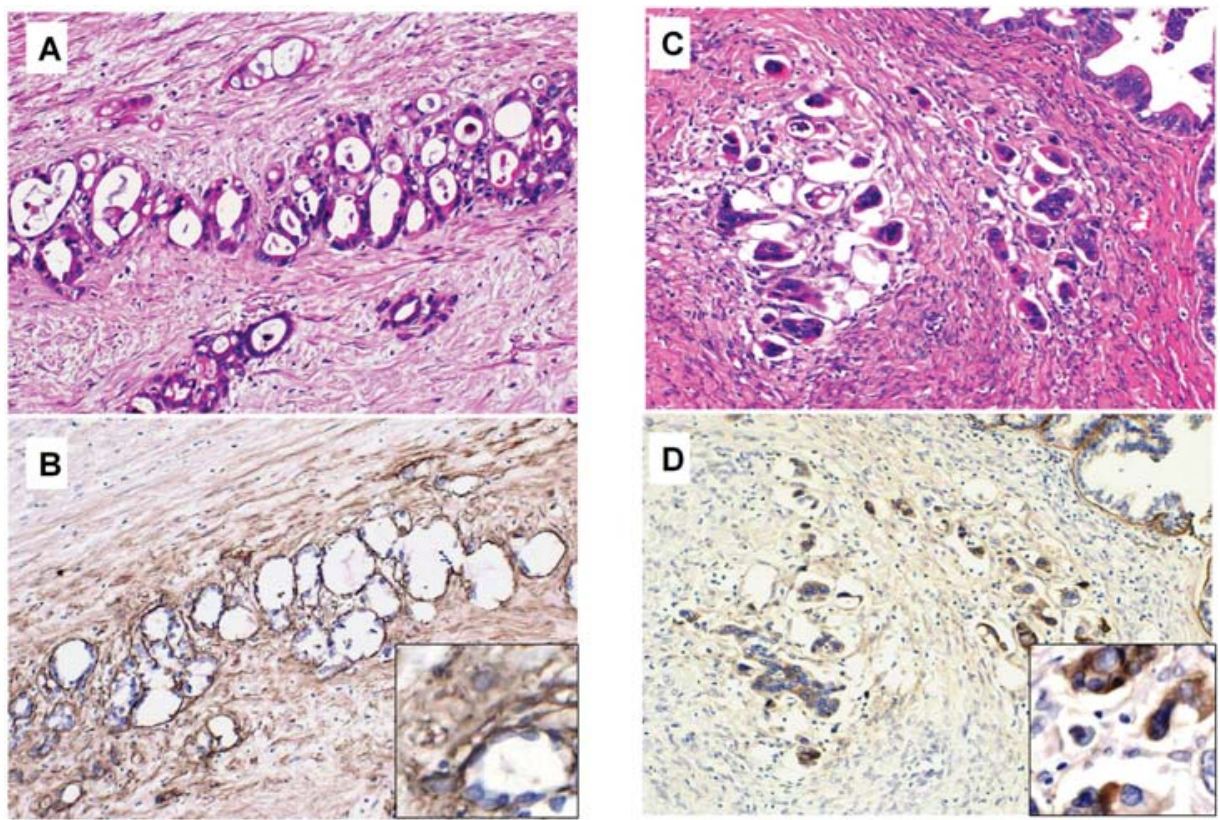

Figure 3. Hematoxylin-eosin findings and laminin $\gamma 2$ immnohistochemical results of infiltrative invasive lesion. (A) Well differentiated invasive lesion, HE, x100. (B) Well differentiated invasive lesion, laminin $\gamma 2$ chain immunostaining, x100; the basement membrane is partly identified by intense staining. The surrounding stromal cells also show immunostaining. The score is BM 2 and S 2. (C) Poorly differentiated infiltrative invasive lesion, HE, x100. (D) Poorly differentiated infiltrative invasive lesion, laminin $\gamma 2$ chain immunostaining, x100; cytoplasmic staining pattern is identified as seen in poorly differentiated microinvasive lesion, the score is CYT 2.

pattern between well and poorly differentiated lesions (Fig. 3B and D). In microinvasion, BM staining was more frequently detected in well differentiated lesions, and CYT staining was more frequently detected in poorly differentiated lesions, although there is no statistically significant difference (Fig. $2 \mathrm{~B}$ and $\mathrm{D})$.
Mural nodules demonstrated similar laminin $\gamma 2$ expression pattern as seen in poorly differentiated infiltrative invasive lesions (Fig. 4B and D). The single case of a mural nodule exclusively composed of a sarcomatoid carcinoma component did not react to laminin $\gamma 2$. 

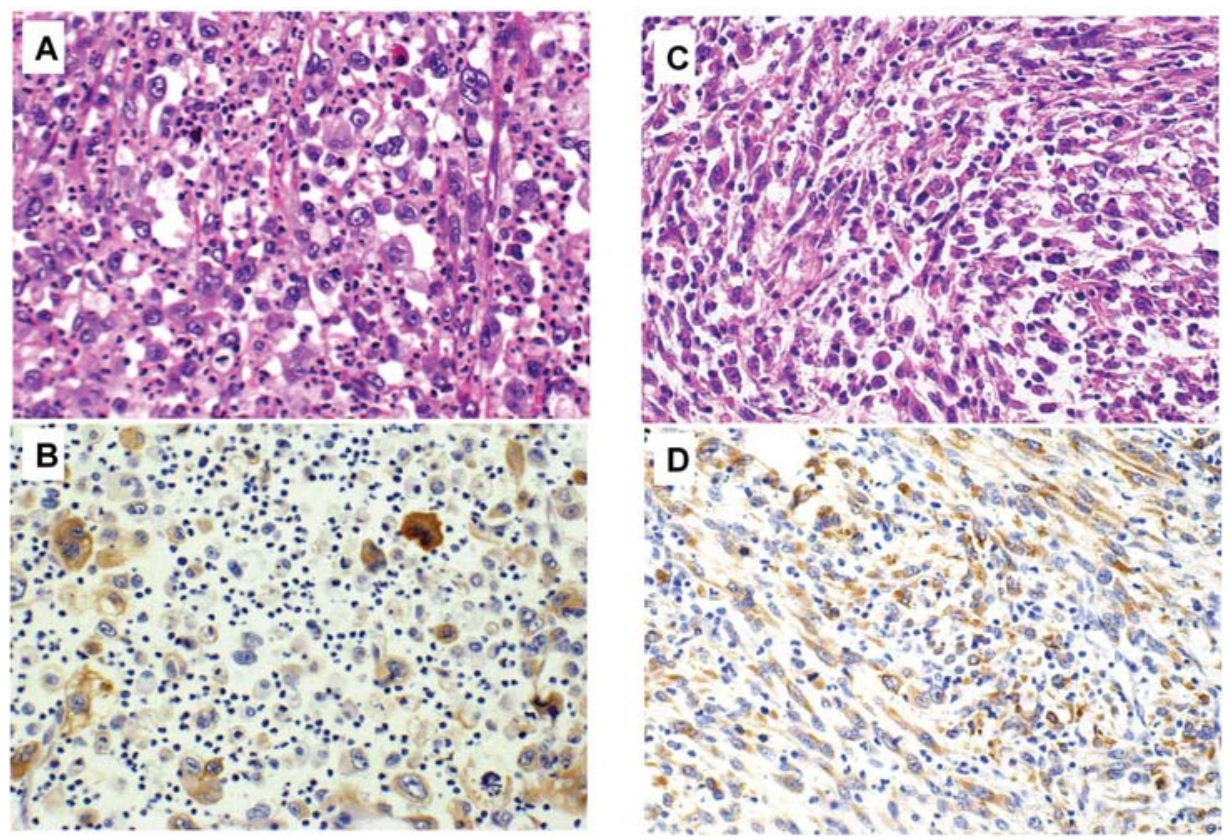

Figure 4. Hematoxylin-eosin findings and laminin $\gamma 2$ immunohistochemical results of mural nodules. (A) Anaplastic carcinoma, HE, x100. (B) Anaplastic carcinoma, laminin $\gamma 2$ chain immunostaining, $x 100$; the score is CYT 2. (C) Sarcomatoid carcinoma, HE, $x 100$. (D) Sarcomatoid carcinoma, laminin $\gamma 2$ chain immunostaining, $\mathrm{x} 100$; the cytoplasm of spindle cells are also stained as seen in anaplastic carcinoma, the score is CYT 2.

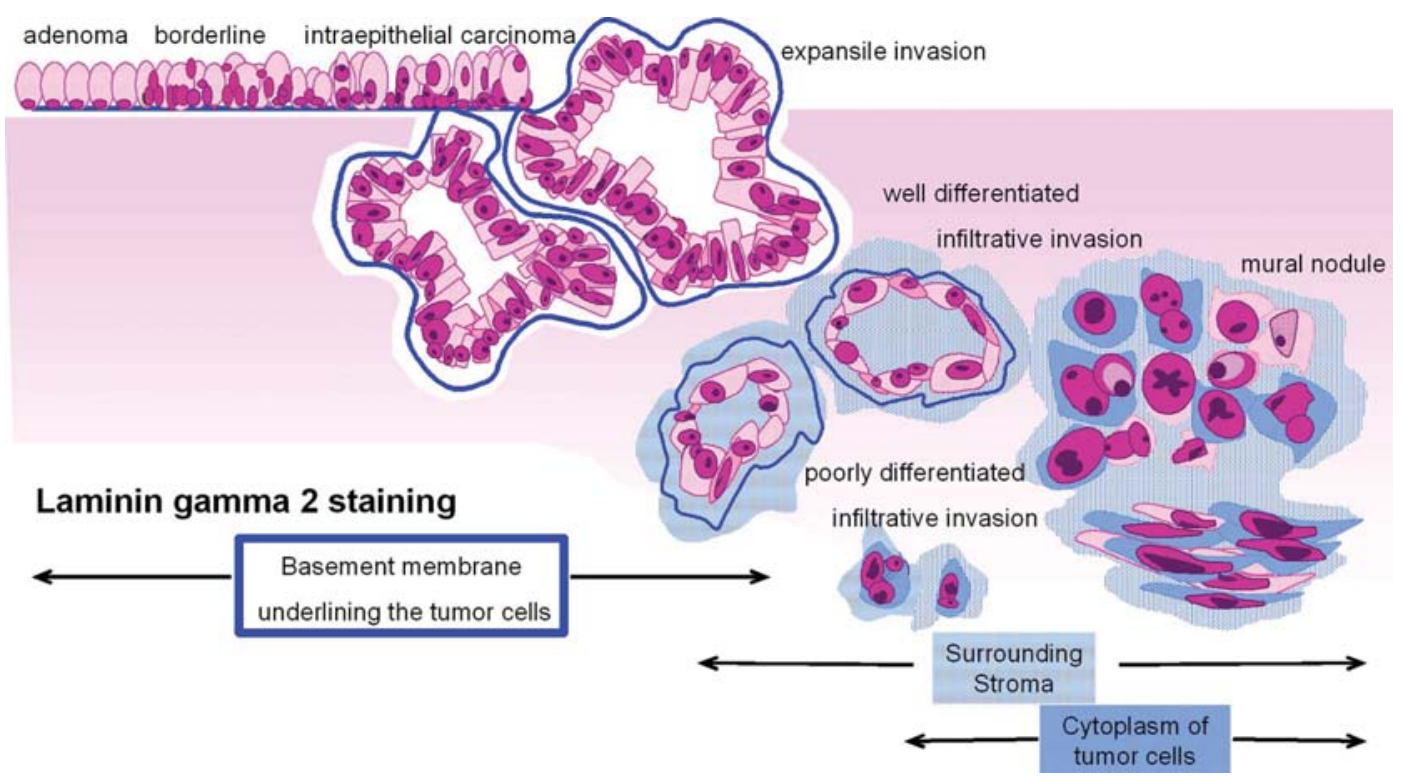

Figure 5. Our progression model of gastro-intestinal type ovarian mucinous neoplasms. As the tumor progress, the nuclear atypia becomes conspicuous. Noninvasive tumor cells exhibit basement membranous accumulation of the laminin $\gamma 2$ chain. Proliferating carcinoma cells start to form expansile invasive carcinoma and then carcinoma cells invade into the surrounding stroma. The invasive nests which maintain the glandular structure represent basement membranous accumulation of the laminin $\gamma 2$ chain, like that seen in expansile invasive carcinoma. Once the glandular formation degrades, the basement membrane of the decrepit and scattered invasive cells become inconspicuous, and laminin $\gamma 2$ becomes to localize in the cytoplasm of tumor cells and the surrounding stroma. The anaplastic/sarcomatoid carcinoma exhibit similar localization of laminin $\gamma 2$ chain to poorly differentiated invasive mucinous carcinoma. Mural nodules composed of anaplastic or sarcomatoid carcinoma cells may develop from infiltrative invasive carcinoma, and laminin $\gamma 2$ may have some influence on that development.

We investigated the relationship between clinicopathological variables and patient prognosis, then only clinical stage had significant relationship with patient prognosis, and the localization of laminin $\gamma 2$ expression did not correlate with patient survival (data not shown).

\section{Discussion}

Subcellular localization of the laminin $\gamma 2$ chain, which is thought to be a tumor invasive promoter, was associated with each phase of mucinous tumor development in the current study. 
Table I. Grading for laminin-5 in each morphological lesion.

\begin{tabular}{|c|c|c|c|c|c|c|c|}
\hline \multirow[t]{2}{*}{ Lesion } & \multirow[t]{2}{*}{ Total } & \multicolumn{2}{|c|}{ BM accumulation } & \multicolumn{2}{|c|}{ CYT accumulation } & \multicolumn{2}{|c|}{$\mathrm{S}$ accumulation } \\
\hline & & $0-1$ & 2 & $0-1$ & 2 & $0-1$ & 2 \\
\hline Adenoma & 55 & $13(24 \%)$ & $42(76 \%)$ & $55(100 \%)$ & 0 & $55(100 \%)$ & 0 \\
\hline Borderline & 60 & $21(35 \%)$ & $39(65 \%)$ & $59(98 \%)$ & $1(2 \%)$ & $60(100 \%)$ & 0 \\
\hline Intraepithelial carcinoma & 17 & $3(18 \%)$ & $14(82 \%)$ & $17(100 \%)$ & 0 & $17(100 \%)$ & 0 \\
\hline Expansile invasive carcinoma & 38 & $16(42 \%)$ & $22(58 \%)$ & $37(97 \%)$ & $1(3 \%)$ & $37(97 \%)$ & $1(3 \%)$ \\
\hline Microinvasion & 20 & $9(45 \%)$ & $11(55 \%)$ & $11(55 \%)$ & $9(45 \%)$ & $19(95 \%)$ & $1(5 \%)$ \\
\hline Infiltrative carcinoma & 19 & $12(63 \%)$ & $7(37 \%)$ & $4(21 \%)$ & $15(79 \%)$ & $10(53 \%)$ & $9(47 \%)$ \\
\hline Mural nodule & 5 & $5(100 \%)$ & 0 & $1(20 \%)$ & $4(80 \%)$ & $4(60 \%)$ & $2(40 \%)$ \\
\hline
\end{tabular}

Table II. Comparison of laminin-5 localization according to the morphological feature.

\begin{tabular}{|c|c|c|c|}
\hline & BM accumulation $2+$ & CYT accumulation $2+$ & S accumulation $2+$ \\
\hline \multicolumn{4}{|l|}{ Invasive lesion } \\
\hline Non-obviously (n=170) & $117(69 \%)$ & $2(1.2 \%)$ & $1(0.6 \%)$ \\
\hline Obviously $(n=24)$ & $7(29 \%)$ & $19(79 \%)$ & $12(50 \%)$ \\
\hline P-value & $0.00037^{\mathrm{a}}$ & $<0.0001^{\mathrm{a}}$ & $<0.0001^{\mathrm{a}}$ \\
\hline \multicolumn{4}{|l|}{ Invasive lesion } \\
\hline Well differentiated $(\mathrm{n}=12)$ & $7(58 \%)$ & $2(17 \%)$ & $8(67 \%)$ \\
\hline Poorly differentiated $(n=15)$ & $1(6.7 \%)$ & $13(87 \%)$ & $4(27 \%)$ \\
\hline P-value & $0.00348^{\mathrm{a}}$ & $0.00027^{\mathrm{a}}$ & 0.09126 \\
\hline \multicolumn{4}{|l|}{ Microinvasion } \\
\hline Well differentiated $(n=18)$ & $11(61 \%)$ & $7(39 \%)$ & $1(5.6 \%)$ \\
\hline Poorly differentiated $(n=6)$ & $2(33 \%)$ & $4(67 \%)$ & $0(0 \%)$ \\
\hline P-value & 0.477960 & 0.47797 & 0.55535 \\
\hline
\end{tabular}

${ }^{\mathrm{a}} \mathrm{p}<0.05$

In the lesions of adenoma, borderline and non-obviously invasive carcinoma, the basement membranous accumulation of laminin $\gamma 2$ was dominant, and this staining pattern was thought to reflect an adhesive characteristic of laminin $\gamma 2$. Laminin-5 stabilizes the epithelial/stromal junction through binding with integrin $\alpha 6 \beta 4$ which forms the hemidesmosome structure (27) with cell-adhesive function. In ovarian mucinous neoplasms without infiltrative invasion, laminin 5 assumes the role to stabilize tumor cells on the basement membrane. On the other hand, the basement membranous structure in the well-differentiated infiltrative invasive carcinoma was maintained, and laminin $\gamma 2$ accumulation was occasionally identified. As for obviously invasive carcinoma, the association between loss of basement membrane continuity and increasing tumor malignancy has been commonly accepted, but continuous basement membrane structures have been found in some invasive carcinoma (31). In a previous study, basement membranes were reported not only to be degraded, but also synthesized in carcinoma (32). It may explain why basement membranes were recognized in welldifferentiated infiltrative invasive carcinoma.

The stromal accumulation was predominantly observed in well-differentiated infiltrative invasion, anaplastic carcinoma and sarcomatoid carcinoma. In invasive intrahepatic cholangiocarcinoma, the stromal accumulation of laminin $\gamma 2$ were significantly correlated with tumor aggressive factors and poor prognosis (19). The stromal laminin $\gamma 2$ expression is thought to contribute to the tumor cell migration and invasion in a variety of carcinomas $(18,19,34)$. The $\gamma 2$ chain which expressed in the stroma can be explained as below. The hemidesmosome structure can be disrupted by tyrosine phosphorylation of $B 4$ integrin, and this is induced by epidermal growth factor (EGF) (27). Laminin $\gamma 2$ chain was revealed to contain EGF-like domain (28), and once the $\gamma 2$ chain is physiologically processed by some stimulating factors such as matrix metalloproteinase (MMP) or bone morphogenetic protein-1 (BMP-1) $(29,30)$, the EGF-receptor of $\beta 4$ integrin would be stimulated, inducing the disruption of 
hemidesmosome and tumor cell migration. The soluble fragments of the $\mathrm{N}$-terminal region which is produced in this processing, are detected in normal human circulation and recognized as an invasion indicator (35). In well differentiated infiltrative invasive carcinoma, various tumor invasion stimulators such as MMP or BMP-1 may function, then fragments of $\gamma 2$ chain may express in the tumor stroma. The resultant laminin 5 molecule would be stabilized in the extracellular matrix (36), and this can explain the basement membranous $\gamma 2$ chain identified in well differentiated infiltrative invasive carcinoma.

Cytoplasmic accumulation of laminin $\gamma 2$ chain was predominantly identified in poorly differentiated infiltrative invasive carcinoma, anaplastic carcinoma and sarcomatoid carcinoma, and cytoplasmic expression of laminin $\gamma 2$ was reported in various invasive carcinoma. In colorectal carcinoma, cytoplasmic 'pseudo-fragments' were immunohistochemically positive with laminin $\gamma 2$ (33). In invasive gastric carcinoma, the cytoplasmic expression of laminin $\gamma 2$ was also detected in budding cells or dissociating cells (18). The authors demonstrated that the cytoplasmic laminin $\gamma 2$ was secreted by gastric carcinoma cells themselves in the form of $\gamma 2$ monomer, which was thought to have some biological activities to promote tumor cell invasion. Also in ovarian mucinous neoplasms, it is suggested that aggressive tumor cells may secrete laminin $\gamma 2$ chain, and it may accumulate in tumor cytoplasms, and stimulate carcinoma cells to migrate and scatter into the surrounding stroma as well as the primary gastro-intestinal carcinoma. Laminin $\gamma 2$ chain is expected to change the characteristics from adhesive to invasive one, as its localization shifts from in basement membrane to in cytoplasm or around the stroma.

In our cases, mural nodule cases demonstrated anaplastic carcinoma or sarcomatoid carcinoma, and the predominant accumulation patterns of laminin $\gamma 2$ were the CYT and S patterns, similarly to that seen in infiltrative invasive carcinoma. This result gave further support to our presumption that certain mural nodules which represent anaplastic or sarcomatoid carcinoma, may be included in the mucinous sequential process, and may develop from adenoma or borderline tumor. Our proposed progression model of ovarian mucinous neoplasm is illustrated in Fig. 5.

In conclusion, cytoplasmic and stromal accumulation of laminin $\gamma 2$ chain in mucinous ovarian neoplasms closely related to destructive stromal invasion of carcinoma cells. Although the correlation with patient prognosis is unclear, there is a possibility that laminin $\gamma 2$ chain may function as a promoter of tumor migration or invasion in ovarian mucinous neoplasms.

\section{References}

1. Siedman JD, Russell P and Kurman RJ: Surface epithelial tumor of the ovary. In: Blaustein's Pathology of the Female Genital Tract. 5th edition, Kurman RJ (eds). Springer-Verlag, New York, pp810-811, 2002

2. Lee KR and Scully RE: Mucinous tumors of the ovary: a clinicopathologic study of 196 borderline tumors (of intestinal type) and carcinomas, including an evaluation of 11 cases with 'pseudomyxoma peritonei'. Am J Surg Pathol 24: 1447-1464, 2000.

3. Puls LE, Powell DE, DePriest PD, et al: Transition from benign to malignant epithelium in mucinous and serous ovarian cystadenocarcinoma. Gynecol Oncol 47: 53-57, 1992.
4. Riopel MA, Ronnett BM and Kurman RJ: Evaluation of diagnostic criteria and behavior of ovarian intestinal-type mucinous tumors: atypical proliferative (borderline) tumors and intraepithelial, microinvasive, invasive, and metastatic carcinomas. Am J Surg Pathol 23: 617-635, 1999.

5. Hoerl HD and Hart WR: Primary ovarian mucinous cystadenocarcinomas: a clinicopathologic study of 49 cases with long-term follow-up. Am J Surg Pathol 22: 1449-1462, 1998

6. Rodriguez IM and Prat J: Mucinous tumors of the ovary: a clinicopathologic analysis of 75 borderline tumors (of intestinal type) and carcinomas. Am J Surg Pathol 26: 139-152, 2002.

7. Ronnett BM, Kajdacsy-Balla A, Gilks CB, et al: Mucinous borderline ovarian tumors: points of general agreement and persistent controversies regarding nomenclature, diagnostic criteria, and behavior. Hum Pathol 35: 949-960, 2004

8. Hart WR: Mucinous tumors of the ovary: a review. Int $\mathbf{J}$ Gynecol Pathol 24: 4-25, 1994.

9. Baergen RN and Rutgers JL: Mural nodules in common epithelial tumors of the ovary. Int J Gynecol Pathol 13: 62-72, 1994.

10. Chan YF, Ho HC, Yau SM, et al: Ovarian mucinous tumor with mural nodules of anaplastic carcinoma. Gynecol Oncol 35: $112-119,1989$.

11. Hayman JA and Ostor AG: Ovarian mucinous tumour with a focus of anaplastic carcinoma: a case report. Pathology 17: 591-593, 1985.

12. Nakamura E, Shimizu M, Mikami Y, et al: Ovarian mucinous cystadenocarcinoma with malignant mural nodules. Pathol Int 48: 645-648, 1998

13. Nichols GE, Mills SE, Ulbright TM, et al: Spindle cell mural nodules in cystic ovarian mucinous tumors. A clinicopathologic and immunohistochemical study of five cases. Am J Surg Pathol 15: 1055-1062, 1991

14. Beck K, Hunter I and Engel J: Structure and function of laminin: anatomy of a multidomain glycoprotein. FASEB J 4: 148-160, 1990.

15. Mizushima H, Koshikawa N, Moriyama K, et al: Wide distribution of laminin-5 gamma 2 chain in basement membranes of various human tissues. Horm Res 50 (Suppl 2): 7-14, 1998.

16. Pyke C, Romer J, Kallunki P, et al: The gamma 2 chain of kalinin/laminin 5 is preferentially expressed in invading malignant cells in human cancers. Am J Surg Pathol 145: 782$791,1994$.

17. Pyke C, Salo S, Ralfkiaer E, et al: Laminin-5 is a marker of invading cancer cells in some human carcinomas and is coexpressed with the receptor for urokinase plasminogen activator in budding cancer cells in colon adenocarcinomas. Cancer Res 55: 4132-4139, 1995.

18. Koshikawa N, Moriyama $\mathrm{K}$, Takamura $\mathrm{H}$, et al: Overexpression of laminin gamma 2 chain monomer in invading gastric carcinoma cells. Cancer Res 59: 5596-5601, 1999.

19. Aishima S, Matsuura S, Terashi T, et al: Aberrant expression of laminin gamma 2 chain and its prognostic significance in intrahepatic cholangiocarcinoma according to growth morphology. Mod Pathol 17: 938-945, 2004.

20. Moriya Y, Niki T, Yamada T, et al: Increased expression of laminin-5 and its prognostic significance in lung adenocarcinomas of small size. An immunohistochemical analysis of 102 cases. Cancer 91: 1129-1141, 2001.

21. Soini Y, Maatta M, Salo S, et al: Expression of the laminin gamma 2 chain in pancreatic adenocarcinoma. J Pathol 180: 290-294, 1996.

22. Patel V, Aldridge K, Ensley JF, et al: Laminin-gamma2 overexpression in head-and-neck squamous cell carcinoma. Int $\mathrm{J}$ Cancer 99: 583-588, 2002.

23. Yamamoto H, Itoh F, Iku S, et al: Expression of the gamma (2) chain of laminin-5 at the invasive front is associated with recurrence and poor prognosis in human esophageal squamous cell carcinoma. Clin Cancer Res 7: 896-900, 2001.

24. Skyldberg B, Salo S, Eriksson E, et al: Laminin-5 as a marker of invasiveness in cervical lesions. J Natl Cancer Inst 91: 1882-1887, 1999.

25. Seidman JD, Kurman RJ and Ronnett BM: Primary and metastatic mucinsou adenocarcinomas in the ovaries: Incidence in routine practice with a new approach to improve intraoperative diagnosis. Am J Surg Pathol 27: 985-993, 2003.

26. Yemelyanova AV, Vang R and Judson K, et al: Distinction of primary and metastatic mucinous tumors involving the ovary: analysis of size and laterality data by primary site with reevaluation of an algorithm for tumor classification. Am J Surg Pathol 32: 128-138, 2008. 
27. Niessen CM, Hogervorst F, Jaspars LH, et al: The alpha 6 beta 4 integrin is a receptor for both laminin and kalinin. Exp Cell Res 211: 360-367, 1994.

28. Schenk S, Hintermann E, Bilban M, et al: Binding to EGF receptor of a laminin-5 EGF-like fragment liberated during MMPdependent mammary gland involution. J Cell Biol 161: 197-209, 2003.

29. Amano S, Scott IC, Takahara K, et al: Bone morphogenetic protein 1 is an extracellular processing enzyme of the laminin 5 gamma 2 chain. J Biol Chem 275: 22728-22735, 2000

30. Veitch DP, Nokelainen P, McGowan KA, et al: Mammalian tolloid metalloproteinase, and not matrix metalloprotease 2 or membrane type 1 metalloprotease, processes laminin-5 in keratinocytes and skin. J Biol Chem 278: 15661-15668, 2003.

31. Flug M and Kopf-Maier P: The basement membrane and its involvement in carcinoma cell invasion. Acta Anat 152: 69-84, 1995
32. Lohi J: Laminin-5 in the progression of carcinomas. Int J Cancer 94: 763-767, 2001

33. Shinto E, Mochizuki H, Ueno H, et al: A novel classification of tumour budding in colorectal cancer based on the presence of cytoplasmic pseudo-fragments around budding foci. Histopathology 47: 25-31, 2005 .

34. Kato N, Sasou S, Teshima S, et al: Overexpression of laminin-5 gamma 2 chain in clear cell carcinoma of the ovary. Virchows Arch 450: 273-278, 2007.

35. Katayama M, Sanzen N, Funakoshi A, et al: Laminin gamma 2chain fragment in the circulation: a prognostic indicator of epithelial tumor invasion. Cancer Res 63: 222-229, 2003.

36. Aumailley M, El Khal A, Knoss N, et al: Laminin 5 processing and its integration into the ECM. Matrix Biol 22: 49-54, 2003. 